This item was submitted to Loughborough's Research Repository by the author.

Items in Figshare are protected by copyright, with all rights reserved, unless otherwise indicated.

\title{
Elementary stratified flows with stability at low Richardson number
}

PLEASE CITE THE PUBLISHED VERSION

https://doi.org/10.1063/1.4904871

PUBLISHER

() AIP Publishing

VERSION

AM (Accepted Manuscript)

PUBLISHER STATEMENT

This work is made available according to the conditions of the Creative Commons Attribution-NonCommercialNoDerivatives 4.0 International (CC BY-NC-ND 4.0) licence. Full details of this licence are available at: https://creativecommons.org/licenses/by-nc-nd/4.0/

\section{LICENCE}

CC BY-NC-ND 4.0

\section{REPOSITORY RECORD}

Barros, Ricardo, and Wooyoung Choi. 2019. "Elementary Stratified Flows with Stability at Low Richardson Number". figshare. https://hdl.handle.net/2134/33836. 


\title{
Elementary stratified flows with stability at low Richardson number
}

\author{
Ricardo Barros ${ }^{1}$ and Wooyoung Choi $^{2}$ \\ ${ }^{1}$ Mathematics Applications Consortium for Science and Industry (MACSI) \\ Department of Mathematics and Statistics \\ University of Limerick \\ Limerick, Ireland \\ ${ }^{2}$ Department of Mathematical Sciences \\ New Jersey Institute of Technology \\ Newark, NJ 07102-1982, USA
}

November 25, 2014

\begin{abstract}
We revisit the stability analysis for three classical configurations of multiple fluid layers proposed by Goldstein [Proc. Roy. Soc. A. 132, 524 (1931)], Taylor [Proc. Roy. Soc. A 132, 499 (1931)] and Holmboe [Geophys. Publ. 24,67 (1962)] as simple prototypes to understand stability characteristics of stratified shear flows with sharp density transitions. When such flows are confined in a finite domain, it is shown that a large shear across the layers that is often considered a source of instability plays a stabilizing role. Presented are simple analytical criteria for stability of these low Richardson number flows.
\end{abstract}

\section{Introduction}

Stability of stratified shear flows have been studied extensively and linear stability analysis has led a number of remarkable results, including Miles-Howard's theorem [1, 2], Howard's semicircle theorem [2], and Synge's generalization of Rayleigh's inflexion point theorem [3].

For given density and velocity profiles, their linear stability characteristics can be determined in a straightforward manner by solving numerically an eigenvalue problem. Nevertheless, it is often cumbersome to solve the eigenvalue problem repeatedly when a large number of physical parameters are involved and, more importantly, it is sometimes difficult to identify the underlying instability mechanisms. Therefore, relatively simple flow configurations for which stability analysis can be performed analytically have sometimes been adopted and the analytic solutions have helped one better understand numerical solutions of the eigenvalue problem for general stratified shear flows.

When the density variation is rather abrupt over a thin vertical distance, it is customary to model the corresponding density profile as fluid layers of different densities. When it is combined with a simple velocity profile such as a linear profile, its stability characteristics can be investigated more conveniently from the analytic solution of the linear stability problem over a wide range of parameters. Goldstein [4] and Taylor [5] indeed considered linear shear flows embedded in three fluid layers in an unbounded domain and discovered that relatively short wavelength disturbances can be unstable even though they are stable in a homogeneous fluid. This contradicts what is commonly believed for the role of stratification since stratification is often assumed to ensure stability. As illustrated originally by Taylor [5] using the simple flow configuration, stratification may lead to instability for a shear flow for which only stationary waves are possible in the homogeneous case. 
Stratification can also alter qualitatively the character of instability of shear flows, as clearly put in evidence by Holmboe [6] for a piecewise linear shear current as the three-layer configuration is modified to the two-layer one. Unlike the homogeneous and three-layer cases, where unstable disturbances travel approximately with the mean velocity (referred to as the Kelvin-Helmholtz instability), two unstable waves excited by the Holmboe instability can travel in opposite directions in the two-layer case. Similar observations were made later experimentally $[7,8]$ and numerically $[9,10]$ for continuously stratified flows. Therefore, simple flow configurations, such as those considered by Goldstein [4], Taylor [5] and Holmboe [6] could serve prototypes to explain the role of stratification in shear flows.

When a fluid domain is bounded by rigid boundaries, the thickness of the domain becomes another important parameter, but its effects are sometimes neglected in stability analysis. From numerical solutions of the linear stability problem in a finite domain, there seems to be a general agreement [9] that, as the top and bottom boundaries are moved in from infinity, relatively short waves that are unstable in an unbounded domain are stabilized although long waves could become unstable. As the two boundaries are moved closer, it could happen that the long waves become stabilized again and the flow could be stable to disturbances of arbitrary wavenumber.

In fact, it was previously shown in [11, figure 4] and [12, the bottom panels of figure 3] that such scenario can happen at low Richardson numbers, defined by $-g \rho^{\prime} / \rho U^{\prime 2}$, where $g$ is the gravitational acceleration, $\rho(z)$ is the density, $U(z)$ is the mean horizontal velocity, and the prime represents differentiation with respect to the vertical coordinate $z$. On the other hand, this does not always remain true at intermediate values of the Richardson number. This seems to be counterintuitive. In general, a continuously stratified shear flow at low Richardson number due to a large velocity gradient is expected to be unstable when the Richardson number is less than a quarter although this condition is a necessary one for instability. This rather surprising stability feature of low Richardson number flows in a finite fluid domain has not been fully explored yet and it is the topic of this paper.

Here we consider three elementary flows proposed by Goldstein [4], Taylor [5] and Holmboe [6] to provide a better understanding of this peculiar stability feature of low Richardson number flows. With recourse to the theory of plane algebraic curves, as used previously by Taylor [5], explicit criteria for stability are obtained. In the case of small density variations for which the Boussinesq approximation has been often adopted, it has been shown [13] that great care should be taken to ensure validity of a simplified analysis based on such approximation. Therefore, the classical Boussinesq approximation is also questioned in capturing the stability feature of low Richardson number flows.

\section{Formulation}

The stability of an inviscid, incompressible, stratified shear flow depends upon the vertical variation of density $\rho(z)$ and the mean horizontal velocity $U(z)$. The behavior of a small two-dimensional, monochromatic disturbance of wavenumber $k$ and wave speed $c$ is governed [14] by

$$
\phi^{\prime \prime}+\frac{\rho^{\prime}}{\rho}\left(\phi^{\prime}-\frac{U^{\prime}}{U-c} \phi\right)+\left[-\frac{g \rho^{\prime}}{\rho(U-c)^{2}}-\frac{U^{\prime \prime}}{U-c}-k^{2}\right] \phi=0,
$$

where the prime indicates differentiation with respect to $z, g$ is the gravitational acceleration, and $\phi$ is the complex amplitude of the stream function.

To simplify the analysis, piecewise linear velocity and piecewise constant density profiles are often adopted. In the same spirit, we consider the following three flow configurations suggested by Goldstein [4], Taylor [5], and Holmboe [6], who first examined their stability characteristics in an unbounded domain. Here we investigate the effects caused by the presence, at finite distance, of two rigid walls confining the fluid with a special emphasis on the case when a large shear across the layers is present. 
(i) Taylor-Goldstein's configuration

$$
U(z)=\left\{\begin{array}{cll}
u_{2} & \text { if } & h<z<H \\
\frac{u_{2}-u_{1}}{2 h} z+\frac{u_{1}+u_{2}}{2} & \text { if } & -h<z<h \\
u_{1} & \text { if } & -H<z<-h
\end{array} \quad, \quad \rho(z)=\left\{\begin{array}{lll}
\rho_{2} & \text { if } & h<z<H \\
\rho_{3} & \text { if } & -h<z<h \\
\rho_{1} & \text { if } & -H<z<-h
\end{array},\right.\right.
$$

(ii) Taylor's configuration

$$
U(z)=\frac{u_{2}-u_{1}}{2 h} z+\frac{u_{1}+u_{2}}{2}, \quad-H<z<H, \quad \rho(z)=\left\{\begin{array}{lll}
\rho_{2} & \text { if } & h<z<H \\
\rho_{3} & \text { if } & -h<z<h \\
\rho_{1} & \text { if } & -H<z<-h
\end{array},\right.
$$

(iii) Holmboe's configuration

$$
U(z)=\left\{\begin{array}{cll}
u_{2} & \text { if } & h<z<H \\
\frac{u_{2}-u_{1}}{2 h} z+\frac{u_{1}+u_{2}}{2} & \text { if } & -h<z<h \\
u_{1} & \text { if } & -H<z<-h
\end{array} \quad, \quad \rho(z)=\left\{\begin{array}{ccc}
\rho_{2} & \text { if } & 0<z<H \\
\rho_{1} & \text { if } & -H<z<0
\end{array} .\right.\right.
$$

Here we assume that the rigid boundaries are placed equidistantly from $z=0$, but the results can be extended to allow non-equidistant rigid walls.

For these configurations, in each subdomain, where $\rho=$ constant and $U^{\prime \prime}=0$, equation (1) can be solved explicitly as linear combinations of $\exp ( \pm k z)$. Then, at each level $z=z_{0}$, where $\rho(z)$, or $U^{\prime}(z)$ is discontinuous, the continuity of pressure and normal velocity at this surface requires the following jump conditions

$$
\llbracket \rho\left[(U-c) \phi^{\prime}-\left(U^{\prime}+\frac{g}{U-c}\right) \phi\right] \rrbracket=0, \quad \llbracket \phi \rrbracket=0,
$$

respectively. Here we have used $\llbracket \rrbracket$ to denote a jump across the interface. By imposing these jump conditions along with no flux conditions at the rigid boundaries, the dispersion relation between the wave speed $c$ and the wavenumber $k$ is then obtained as a polynomial equation (of degree 4) for $c$.

In practice, however, the coefficients of the resulting polynomial equation are complex enough to make rather difficult the task of fully describing the stability features of these flows. To avoid this difficulty, following Taylor [5] and Ovsyannikov [15], we adopt a geometrical approach, where this polynomial equation in a single variable $c$ is interpreted as a plane algebraic curve, which is found effective to study stability of these flows.

\section{Taylor-Goldstein's configuration}

\subsection{Stability analysis for a bounded domain}

With introducing variables $p$ and $q$ defined by

$$
p=\left(u_{1}-c\right) / \sqrt{2 g h}, \quad q=\left(u_{2}-c\right) / \sqrt{2 g h},
$$

the eigenvalue equation for $c$ resulting from (5), together with the boundary conditions, can be written as an algebraic curve of degree 4 on the $(p, q)$-plane:

$$
\left(\beta_{1} p^{2}+\gamma_{3} p q+\gamma_{3}-1\right)\left(\beta_{2} q^{2}+p q+\gamma_{2}-1\right)=\gamma_{3} \beta_{3} p^{2} q^{2}
$$

The coefficients $\beta_{i}$ in the expression are defined by

$$
\begin{gathered}
\beta_{1}=\alpha \operatorname{coth}[\alpha(r-1) / 2]+\gamma_{3}(\alpha \operatorname{coth} \alpha-1), \\
\beta_{2}=\gamma_{2} \alpha \operatorname{coth}[\alpha(r-1) / 2]+\alpha \operatorname{coth} \alpha-1, \quad \beta_{3}=\alpha^{2} \operatorname{csch}^{2} \alpha,
\end{gathered}
$$




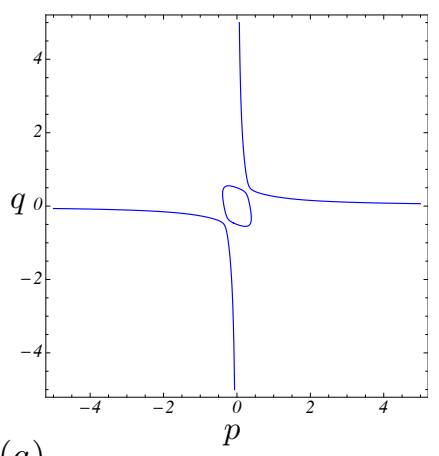

(a)

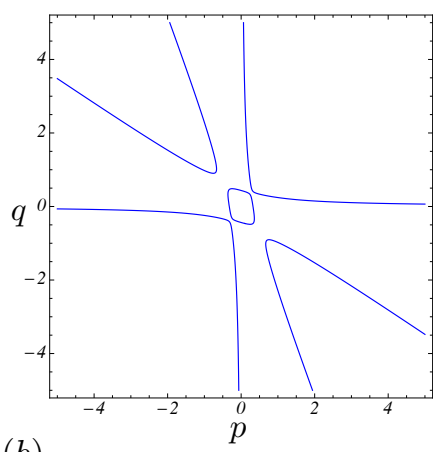

$(b)$

Figure 1: Plots on the $(p, q)$-plane of the curve defined by equation (7) for $r=2, \gamma_{2}=2 / 3$, and $\gamma_{3}=3 / 4$. (a) $\alpha=0.1,(b) \alpha=1.0$.

where $\alpha=2 k h$ is the dimensionless wavenumber, $r=H / h(>1)$ is the depth ratio, and $\gamma_{2}=\rho_{2} / \rho_{3}(<$ 1) and $\gamma_{3}=\rho_{3} / \rho_{1}(<1)$ are the density ratios. Hereafter, we assume that $\gamma_{2}, \gamma_{3}$ are strictly positive, and $r<\infty$, unless clearly stated otherwise, which will be referred to as "in general" when making an assertion.

Then, as shown by Taylor [5], the stability problem is equivalent to finding the intersection between the curve described by (7) and the line of equation given, from (6), by

$$
q=p+\left(u_{2}-u_{1}\right) / \sqrt{2 g h} .
$$

Notice that from definition (6), $p$ is real if and only if the wave speed $c$ is real. Therefore, the flow is stable if the line (10) intersects the curve (7) at four points, and unstable otherwise ${ }^{1}$. We remark that Taylor (1931) used an equation similar to (7) in the limit of $r \rightarrow \infty$, i.e., for the unbounded domain case.

To determine the number of intersections between the curve (7) and the line (10) as the relative velocity increases, the behavior of an algebraic curve at infinity, described here by the asymptotes at infinity, is important as the $q$-intercept is proportional to the magnitude of the relative velocity.

It is convenient to express the curve equation $(7)$ as $P(p, q) \equiv \sum_{k=0}^{4} P_{k}(p, q)=0$, where $P_{k}(p, q)$ is a homogeneous polynomial in $p$ and $q$ of degree $k$, since the factors of the highest degree polynomial define the slopes of the asymptotes to the curve. In the three examples to be considered in this paper, no odd degree polynomials show up in the curve equation. This leads to symmetry about the origin, so that $P(-p,-q)=P(p, q)$, and allows us to use the following results (Primrose [16], Theorem 2, pp. 7-8): ( $i$ ) any simple factor $a p+b q$ of $P_{4}(p, q)$ will have associated an asymptote to the curve, defined by the equation $a p+b q=0 ;($ ii $)$ if $a p+b q$ is a repeated factor of $P_{4}(p, q)$, so that $P_{4}(p, q)=(a p+b q)^{2} Q(p, q)$, then it will have associated at most two possible asymptotes $a p+b q=t_{0}$, where $t_{0}$ is a real root of $Q(b,-a) t^{2}+P_{2}(b,-a)=0$. In both cases, using homogeneous coordinates, $(b,-a, 0)$ is a point at infinity. However, only in $(i i)$ the point $(b,-a, 0)$ is a singular point (see Appendix C). As a result, nonlinear branches at infinity do not exist and the behavior of the curve at infinity is hence completely described by its asymptotes, as mentioned earlier.

For Taylor-Goldstein's configuration, the highest degree polynomial $P_{4}(p, q)$ is given by

$$
P_{4}(p, q) \equiv p q\left[\beta_{1} p^{2}+\left\{\beta_{1} \beta_{2}+\gamma_{3}\left(1-\beta_{3}\right)\right\} p q+\gamma_{3} \beta_{2} q^{2}\right]
$$

\footnotetext{
${ }^{1}$ To be more precise, the flow is stable whenever the number of intersection points is equal to the degree of the polynomial equation for the wave speed $c$. In general, as explained in $\S 2$, four points are to be expected. However, in degenerate cases, this number could be reduced (see Appendix A).
} 

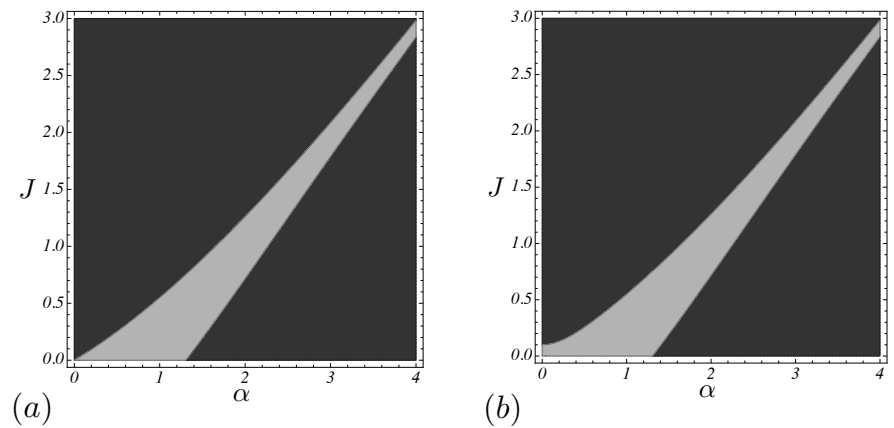

(b)

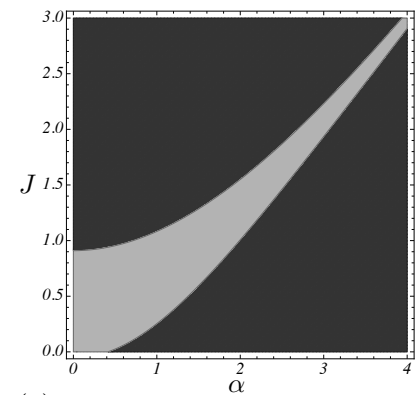

(c)

Figure 2: Stability diagrams on the $(\alpha, J)$-plane for Taylor-Goldstein's configuration with $\gamma_{2}=2 / 3$ and $\gamma_{3}=3 / 4$. The total depth ratios are $r=\infty, 10$, and 2 (from left to right). The dark shaded region corresponds to a stable region with four real roots and the light shaded region corresponds to the unstable region with two complex and two real roots. Hereafter, the same color scheme will be adopted.

from which it is found that, regardless of parameters, the curve (7) has two asymptotes: $p=0$ and $q=0$. It can be further proven that the asymptote $p=0$ for large negative values of $q$ can only be connected to the asymptote $q=0$ for large negative values of $p$ in the third quadrant. By symmetry, such a real connected component should also exist in the first quadrant (cf. figure 1). As a consequence, the intersection between the curve and the line can never be empty. Moreover, the curve cuts the axes at four points close to the origin. These points belong to the contour represented in figure 1 by an oval, which guarantees stability of the flow with a small relative velocity between the layers (i.e. small $\left|u_{2}-u_{1}\right| / \sqrt{2 g h}$ ), as the line given by equation (10) with a small intercept will cut the curve at four distinct points.

To examine if there are any additional asymptotes to the curve, we have to take into account the terms in the bracket in (11), and consider the following quadratic equation for $v \equiv p / q$ :

$$
\beta_{1} v^{2}+\left[\beta_{1} \beta_{2}+\gamma_{3}\left(1-\beta_{3}\right)\right] v+\gamma_{3} \beta_{2}=0 .
$$

The discriminant of this quadratic equation fully determines the number of real roots and, when positive, two extra asymptotes to the curve exist. Since $\beta_{1}>0, \beta_{2}>0$, and $0<\beta_{3} \leqslant 1$, it follows from Viète's formulas that, if (12) has two distinct real roots, both of them are negative. This means that the associated asymptotes have negative slopes, and lie therefore on the second and fourth quadrants. Figure 1(b) supports such scenario and suggests that, in the presence of such asymptotes, stability of a flow with a very large relative velocity is possible.

Figure 1 shows two of possible scenarios obtained for two different sets of parameters. To fully describe the planar curve, a rather lengthy discussion is needed; thus, the details are left to the Appendices, and it will be simply noted here that the two configurations presented in figure 1 are, in general, the only possible scenarios.

This geometrical approach is of course equivalent to the standard analytical approach, where the quartic equation for the phase velocity $c$ is obtained by substituting (6) into (7). Then, using Fuller's root location criteria $[17,18,19]$, the stability diagram on the $(\alpha, J)$-plane can be drawn, as shown in figure 2, where the Richardson number $J$ is defined for this configuration by

$$
J=\left(\frac{\rho_{1}-\rho_{2}}{\rho_{3}}\right) \frac{2 g h}{\left(u_{1}-u_{2}\right)^{2}} .
$$

As shown in figure 2, for any fixed Richardson number $J$, there is always a limited range of wavenumbers for which a single unstable wave appears. This band of wavenumber for instability 
becomes narrower as $J$ increases. As the depth ratio $r$ decreases, the boundary effects can be perceived by a destabilizing effect on the long waves as the upper branch of the stability boundary rises. On the other hand, the shorter waves become more stable as the lower branch of the stability boundary moves to the left. Such phenomenon was reported for continuously stratified flows in the numerical work of Hazel [9].

From this observation, one can further imagine that, in a bounded domain with certain physical parameters, the lower branch of the stability boundary could move further to the left so that it actually starts to rise along the $J$-axis. For such parameters, the three-layer configuration would then become stable for small values of $J$, or large values of $\left|u_{1}-u_{2}\right| / \sqrt{2 g h}$, while remaining unstable for large and intermediate Richardson number. This observation has not been fully explored yet and the condition under which it is valid has not been known ${ }^{2}$.

\subsection{A condition for stability for arbitrarily low Richardson number flows}

For Taylor-Goldstein's configuration at low Richardson number to be stable, it is necessary and sufficient that two extra asymptotes at infinity, governed by equation (12), exist for all $\alpha$.

As a first step, we consider the long wave limit of equation (12), which can be reduced, as $\alpha \rightarrow 0$, to

$$
v^{2}+\left(\frac{2 \gamma_{2}}{r-1}\right) v+\gamma_{2} \gamma_{3}=0
$$

where $v=p / q$. Distinct real roots for this equation ensure the existence of such additional asymptotes. Multiple roots could, in principle, also provide the asymptotes, but it can be shown that this scenario does not apply here. This implies that in order for this stability feature to hold, it is necessary that

$$
\gamma_{3} / \gamma_{2}<1 /(r-1)^{2}
$$

Although the condition given by (15) is valid as $\alpha \rightarrow 0$, a further inspection of the stability diagram seems to indicate that it should suffice for any values of $\alpha$, relying on the monotonic behavior of stability boundaries as functions of the wavenumber $\alpha$. In fact, it can be shown that the discriminant of equation (12) under the same condition as (15) becomes positive and has two distinct real roots for any values of $\alpha$ by observing $\beta_{1} \geqslant 2 /(r-1)$ and $\beta_{2} \geqslant 2 \gamma_{2} /(r-1)$ for fixed parameters $r, \gamma_{2}$, and $\gamma_{3}$. This proves that the condition (12) is not only necessary, but also sufficient for four asymptotes to exist and, therefore, stability.

This is the main result of the section: the three-layer system is stable for small values of $J$, i.e., $0 \leqslant J \leqslant J_{s}$, when inequality (15), or, equivalently, in dimensional form, $\rho_{3}{ }^{2} /\left(\rho_{1} \rho_{2}\right)<h^{2} /(H-h)^{2}$ is satisfied. Such examples are shown in figures 3 and 4 .

It should be mentioned that, if the the top and bottom boundaries are placed at arbitrary levels $z=H_{2}$ and $z=-H_{1}$, then the stability criterion (15) should be replaced by $\gamma_{3} / \gamma_{2}<\left[\left(r_{1}-1\right)\left(r_{2}-1\right)\right]^{-1}$, with each depth ratio $r_{i}$ defined by $r_{i}=H_{i} / h$.

\subsection{Boussinesq approximation}

It is of interest to examine if a similar stability condition as (15) can be obtained with the Boussinesq approximation, which has been widely adopted in stability analysis for stratified shear flows. Following Goldstein [4], when we assume $\rho_{1,2}=\rho_{0}(1 \pm \varepsilon)$, with $0<\varepsilon<1$, and $\rho_{3}=\rho_{0}$, the stability criterion (15) reads

$$
\varepsilon^{2}<1-(r-1)^{2}
$$

\footnotetext{
${ }^{2}$ Notice that such stability feature would yield, in the non-dispersive limit $(\alpha \rightarrow 0)$, two disconnected regions of stability, characterized respectively by a small/large shear across the layers. This peculiar scenario has been found earlier in hyperbolic models for multilayer flows $[15,19,20]$, but only in the absence of linear shear.
} 

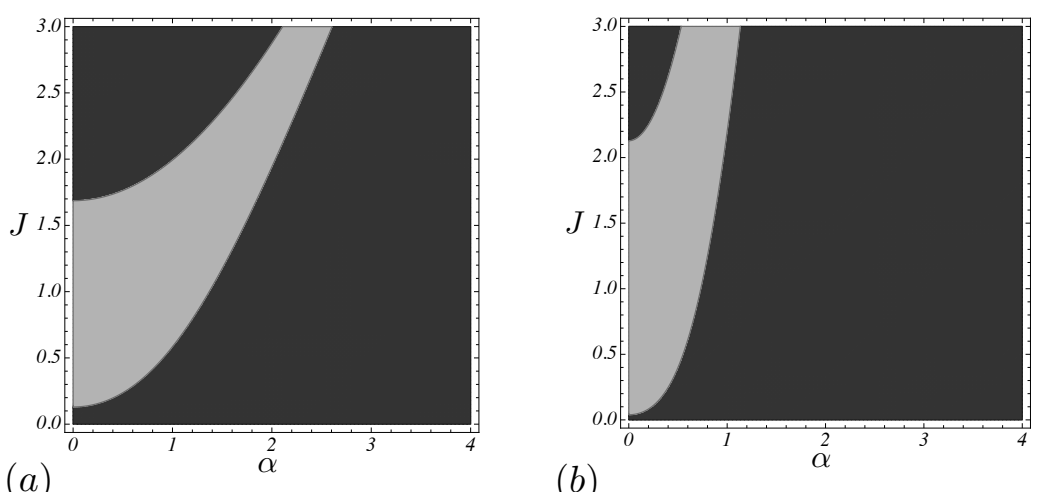

(b)

Figure 3: Stability diagrams on the $(\alpha, J)$-plane for Taylor-Goldstein's configuration for different parameters. (a) $r=2, \gamma_{2}=0.998$, and $\gamma_{3}=0.8$. (b) $r=4, \gamma_{2}=0.99$, and $\gamma_{3}=0.1$. Dark shaded: stable region; Light shaded: unstable region.
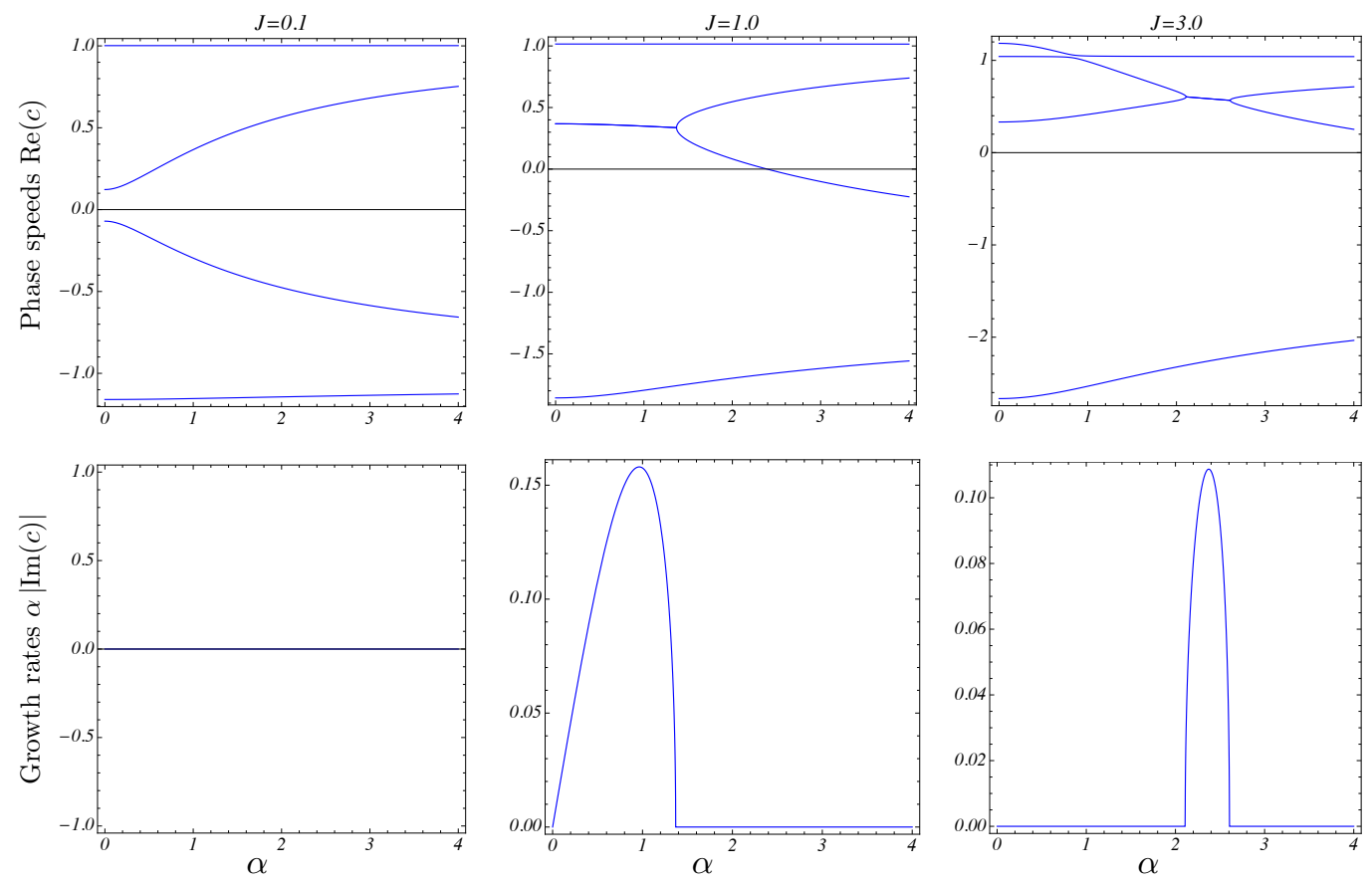

Figure 4: Phase speeds (top panel) and growth rates (bottom panel) for three different Richardson numbers for Taylor-Goldstein's configuration. The physical parameters considered here are the same as in figure $3(a): r=2, \gamma_{2}=0.998$, and $\gamma_{3}=0.8$. 
This shows that Taylor-Goldstein's configuration at low Richardson number is stable only if $r<2$ for this particular stratification.

The classical Boussinesq limit can be obtained by taking the limit of $\varepsilon \rightarrow 0$ in such a way that the Richardson number $J$, defined by $J=4 \varepsilon g h /\left(u_{1}-u_{2}\right)^{2}$, remains finite, or, equivalently, the Froude number defined by $\mathcal{F}=\left|u_{1}-u_{2}\right| /(2 g h)^{1 / 2}$ is small such that $\mathcal{F}=O\left(\varepsilon^{1 / 2}\right)$. The requirement on the magnitude of the shear across the layers indicates that the classical Boussinesq limit to (16) should be taken with care.

From $\gamma_{2}=1-\varepsilon, \gamma_{3}=1-\varepsilon+O\left(\varepsilon^{2}\right), \beta_{1}=\beta+O(\varepsilon)$, and $\beta_{2}=\beta+O(\varepsilon)$, where $\beta$ is defined in (8) with $\gamma_{3}=1$, the Boussinesq approximation yields the algebraic curve given by

$$
\left(\beta p^{2}+p q-\varepsilon\right)\left(\beta q^{2}+p q-\varepsilon\right)=\beta_{3} p^{2} q^{2},
$$

where we have assumed $p=O(q)=O\left(\varepsilon^{1 / 2}\right)$ from the fact that $\mathcal{F}=O\left(\varepsilon^{1 / 2}\right)$. Since the highest degree polynomial $P_{4}(p, q)$ of (17) is identical to (11) with $\gamma_{2}=\gamma_{3}=1$ and is independent of $\varepsilon$, it is identical to that for the homogeneous fluid case for which the configuration is known to be stable for any depth ratio $r$ that lies in $1<r<2$ (Rayleigh [21], vol. II, p. 388). Therefore, the flow in Taylor-Goldstein's configuration under the Boussinesq approximation is stable for large shear if and only if $1<r<2$.

Although this result is consistent with (16) for small $\varepsilon$, we should remark that (17), valid for small $p$ and $q$, should not be used to draw a conclusion on the curve behavior for large $p$ and $q$. This shows that care must be taken to interpret the stability characteristics of low Richardson number flows from any analysis based on the Boussinesq approximation.

On a final note, we would like to point out that extra symmetries are obtained under the Boussinesq approximation. The curve (17) is not only symmetric about the origin, as the original curve (7), but is also symmetric with respect to the lines $q=p$ and $q=-p$. As a consequence, the curve can be expressed simply in terms of $p^{2}+q^{2}$ and $p q$, which leads to a biquadratic form on the wave speed relative to the mean flow (see Appendix D).

\section{Results for other configurations}

\subsection{Taylor's configuration}

For Taylor's configuration, the algebraic curve in the $(p, q)$-plane is given by

$$
\left[\left(\beta_{1}+1\right) p^{2}+\left(\gamma_{3}-1\right) p q+\gamma_{3}-1\right]\left[\left(\beta_{2}+\gamma_{2}\right) q^{2}-\left(\gamma_{2}-1\right) p q+\gamma_{2}-1\right]=\gamma_{3} \beta_{3} p^{2} q^{2},
$$

with $\beta_{1}$ through $\beta_{3}$ given in (8)-(9). Once again, by examining the highest degree polynomial, we find that $p=0$ and $q=0$ are the asymptotes to the curve (18). The existence of additional asymptotes depend on the number of real roots of the quadratic equation for $v \equiv p / q$ :

$$
\left(1-\gamma_{2}\right)\left(\beta_{1}+1\right) v^{2}+\left[\left(\beta_{1}+1\right)\left(\beta_{2}+\gamma_{2}\right)-\left(\gamma_{2}-1\right)\left(\gamma_{3}-1\right)-\gamma_{3} \beta_{3}\right] v+\left(\gamma_{3}-1\right)\left(\beta_{2}+\gamma_{2}\right)=0 .
$$

Notice that, if real roots exist for this equation, they must have opposite signs. Any line with slope 1 will then intersect the curve at least three times ${ }^{3}$. Therefore, this configuration is stable at large shear if and only if such asymptotes exist.

Contrary to Taylor-Goldstein's configuration, the discriminant here is always greater or equal to zero. Therefore, stability is, in general, expected for Taylor's configuration with a large shear. Caution should be taken on identifying whether multiple roots can, or not, provide the aforementioned

\footnotetext{
${ }^{3}$ In general, there will be four intersection points. However, as shown in figure $5(c)$, it could be the case that one of the asymptotes has slope 1 , and only three intersection points are obtained. In this case, the quartic equation for $c$ degenerates into a cubic, and corresponds thus to a stable scenario (see Appendix B).
} 


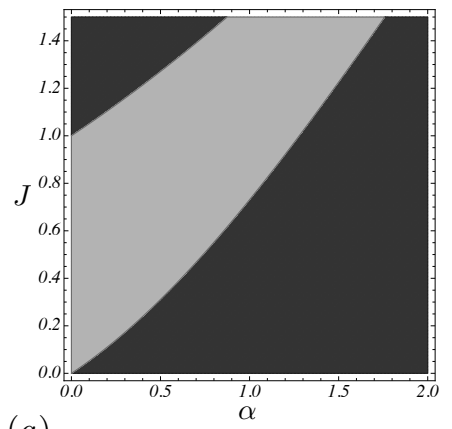

(a)

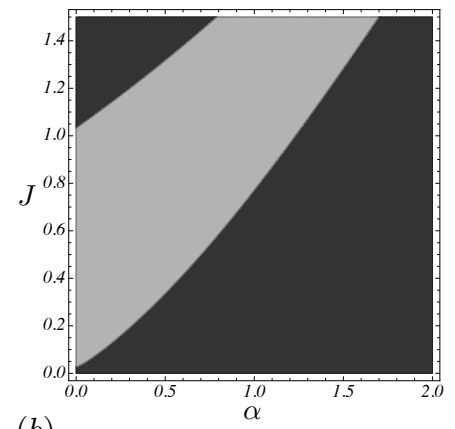

(b)

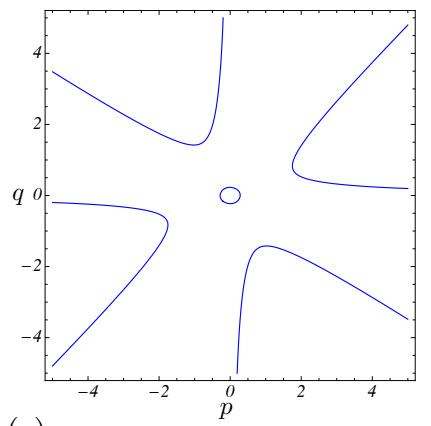

$(c)$

Figure 5: Comparison of stability diagrams for $r=\infty, \gamma_{2}=0.95$, and $\gamma_{3}=0.92$. Dark shaded: stable region; Light shaded: unstable region for Taylor's configuration. (a) with Boussinesq approximation; (b) without Boussinesq approximation; (c) planar curve in the long wave limit $\alpha \rightarrow 0$ supporting the stability result displayed in $(b)$.

asymptotes. However, it is found that discriminant vanishes only for a degenerate case of a twolayer flow $\left(\gamma_{2}=1\right.$ or $\left.\gamma_{3}=1\right)$ with a linear shear current, known to be stable. Hence, in Taylor's configuration, the flow with a large shear is stable for all physical parameters.

We notice that the finite-depth effect is not essential to stabilize this flow with a large shear. This is in great contrast with Taylor-Goldstein's configuration, where, for infinite depth, the upper branch of the stability curve passes through the origin regardless of physical parameters involved (see figure $2(a))$.

We investigate now if the Boussinesq approximation is able to capture such stability feature. Similarly to what was discussed for Taylor-Goldstein's configuration, the Boussinesq limit is described by the curve

$$
\left[(\beta+1) p^{2}-\varepsilon\right]\left[(\beta+1) q^{2}-\varepsilon\right]=\beta_{3} p^{2} q^{2} .
$$

In this case, the highest degree polynomial $P_{4}(p, q)$ is proportional to $p^{2} q^{2}$ and does not have simple factors. By applying Theorem 2(ii) (Primrose [16], pp. 7-8) described in $\S 3.1$, we conclude the existence of parallel vertical (and horizontal) lines of equation $p=t^{ \pm}$(and $q=t^{ \pm}$), where $t^{ \pm}$are distinct real roots of $\left[(\beta+1)^{2}-\beta_{3}\right] t^{2}=\varepsilon(\beta+1)$. An anomaly is produced when $r=\infty$ and $\alpha \rightarrow 0$, for which the higher-order coefficient vanishes and the curve degenerates into a circle of equation $p^{2}+q^{2}=\varepsilon$. This implies that the Boussinesq approximation fails to capture stability of the flow with a large shear when $r=\infty$ (compare figure 5(a) and $(b))$ while it predicts stability for finite $r$.

From this analysis, another limitation of Boussinesq approximation can be perceived. Notice that when $r$ is large enough, $\beta+1 \approx \alpha(1+\operatorname{coth} \alpha)$, and hence the roots $t^{ \pm}= \pm \sqrt{\varepsilon / 2 \alpha}$ become good approximations for the asymptotes $p=t^{ \pm}$(and $q=t^{ \pm}$) to the curve. Unless $\alpha=O(1)$, the assumptions $p=O(q)=O\left(\varepsilon^{1 / 2}\right)$ for $\mathcal{F}=O\left(\varepsilon^{1 / 2}\right)$ can be easily violated. As a result, for large $r$ and small $\alpha$, while the 'internal' modes can be well described quantitatively by the Boussinesq approximation, discrepancies are expected to be found for the 'external' modes (see figure 6).

\subsection{Holmboe's configuration}

Assuming the same background velocity profile as in Talyor-Goldstein's configuration, Holmboe [6] has illustrated how stratification can change qualitatively the character of instability when the thickness of density transition layer vanishes, leading to a two-layer configuration. Along with the classical KelvinHelmholtz instability, a second mode of instability, characterized by two unstable counter propagating waves, should be present. 


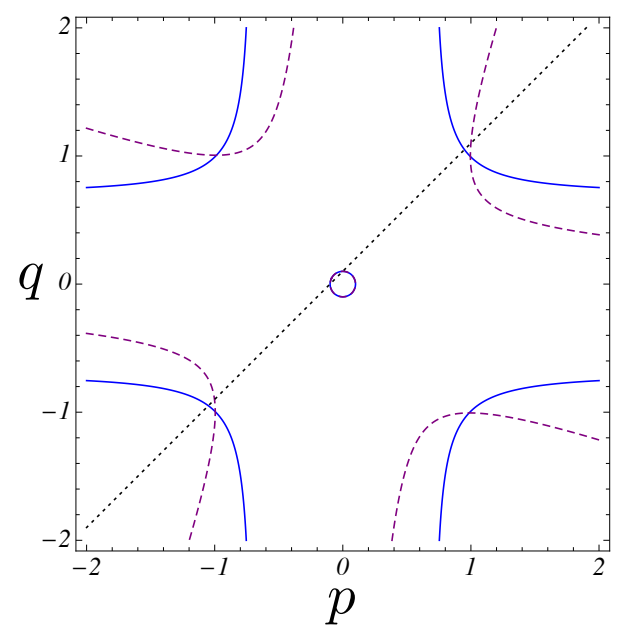

Figure 6: Comparison between the curves with (solid line) and without (dashed line) Boussinesq approximation for Taylor's configuration. The physical parameters considered here are: $\varepsilon=10^{-2}$, $r=200, \alpha=10^{-3}, \gamma_{2}=1-\varepsilon$, and $\gamma_{3}=1 /(1+\varepsilon)$. The dotted line represents the line of equation $q=p+1 / 10$. Notice that the inner components of the two curves are almost indistinguishable, yielding an excellent agreement for two of the roots ('internal' modes) of the dispersion relation. In contrast, the outer components differ substantially, and thus the discrepancies for the 'external' modes.

Here, we will show that a flow at low Richardson number can also be stable for this physical configuration, and a necessary, and possibly sufficient, condition for this to happen shall be presented.

The eigenvalue equation for this configuration can be expressed by the following curve

$$
\left(\lambda_{1} p+q\right)\left[\left(p+\lambda_{1} q\right)\left\{\lambda_{2}(p+q)^{2}+2(\rho-1)\left(2+q^{2}-p^{2}\right)\right\}-\rho \lambda_{3} q(p+q)^{2}\right]=\lambda_{3} p\left(p+\lambda_{1} q\right)(p+q)^{2},
$$

where $\rho=\rho_{2} / \rho_{1}(<1)$ is the density ratio and the new coefficients are defined as follows:

$$
\begin{array}{r}
\lambda_{1}=\alpha[\operatorname{coth}[\alpha(r-1) / 2]+\operatorname{coth}(\alpha / 2)]-1, \\
\lambda_{2}=\alpha(\rho+1) \operatorname{coth}(\alpha / 2), \quad \lambda_{3}=\alpha^{2} \operatorname{csch}^{2}(\alpha / 2) .
\end{array}
$$

As in previous sections, we will describe here what happens in general, leaving thus the degenerate cases left to the Appendix C. This said, let $0<\rho<1$ and $r<\infty$.

For Holmboe waves to exist, four complex values of $c$ should be obtained as solutions of the eigenvalue problem. This could never happen in the three-layer configurations considered previously. Hence, a rather different qualitative behavior of the curve is expected.

In figure 7 , two plots of the curve equation (21) reveal the existence of an eight-shaped curve around the origin, yielding stability of the flow with a small relative velocity between the layers. On the other hand, for intermediate values of $p$ and $q$, there is the possibility of an empty intersection between the curve and the line (10), implying the appearance of two unstable Holmboe waves. We point out that this scenario persists only up to a certain Richardson number ( $c f$. figure 8), and, therefore, Holmboe waves can only be observed for a limited range of values of the Richardson number $J$, defined by $J=4(1-\rho) g h /\left(u_{1}-u_{2}\right)^{2}$. For larger values of $J$, only one-sided instability should be expected $[22,23,13]$.

As before, in order for the flow with a small value of $J$ to be stable to arbitrary disturbances, it is necessary for the curve to have four asymptotes in the long wave limit $(\alpha \rightarrow 0)$, where the highest degree polynomial $P_{4}(p, q)$ of equation (21) is found to be

$$
(v+1)\left(v^{3}+R v^{2}+\rho R v+\rho\right)=0,
$$




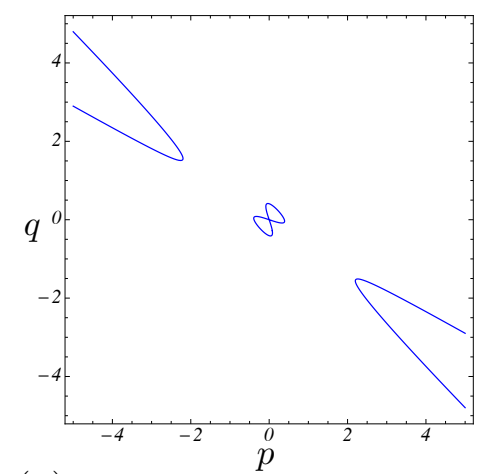

(a)

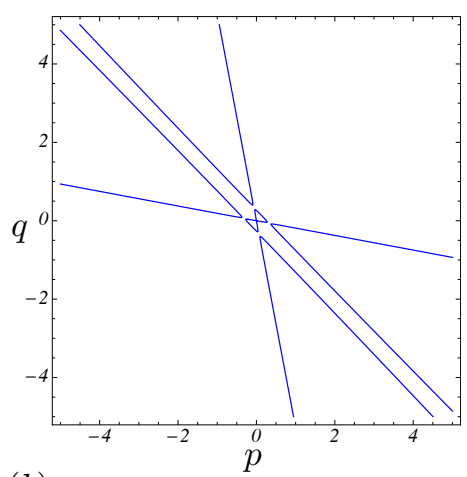

(b)

Figure 7: Plots on the $(p, q)$-plane of the curve defined by equation $(21)$ for $r=2$ and $\rho=0.9$. $(a)$ $\alpha=0.1,(b) \alpha=3.0$.

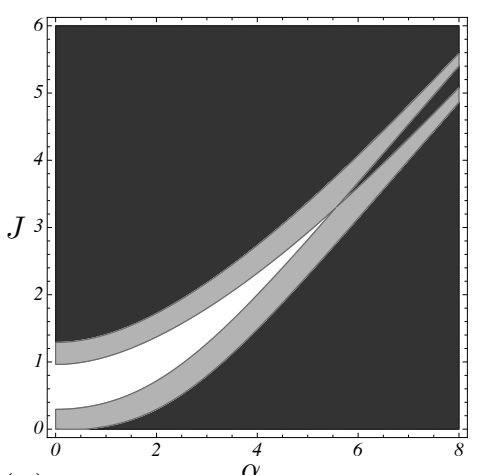

(a)

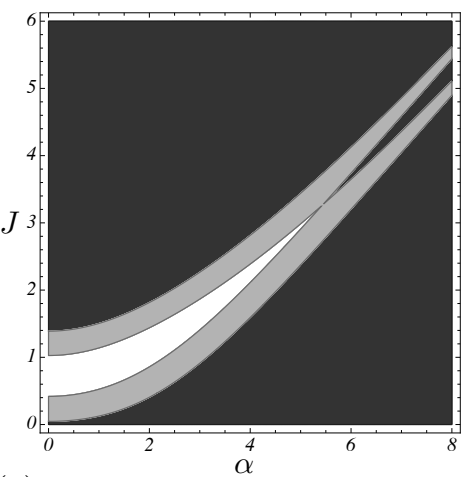

(b)

Figure 8: Stability diagrams on the $(\alpha, J)$-plane for Holmboe's configuration with $\rho=0.7$. $(a) r=1.5$, (b) $r=1.4$. The dark shaded region corresponds to a stable region, the light shaded region corresponds to the unstable region with two complex and two real roots, and the white region corresponds to the unstable region with four complex roots. 


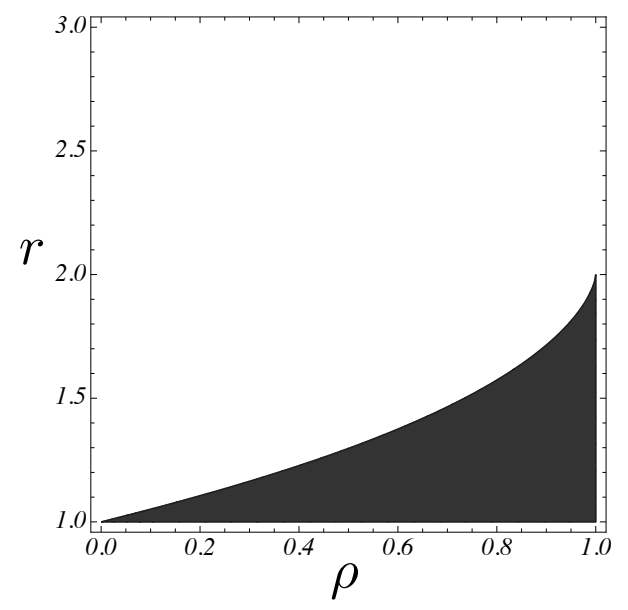

Figure 9: Region on the ( $\rho, r)$-plane where condition (25) holds and Holmboe's configuration is possibly stable at low Richardson number.

with $v=p / q$, and $R$ defined by $R=(r+1) /(r-1)$. It is shown in Appendix $\mathrm{C}$ that $v=-1$ is in fact a simple root of $(24)$, from which it follows $p+q=0$ is an asymptote to the curve in the long wave limit, which remains true for any other values of $\alpha$, as shown in figure 7 .

On the other hand, if multiple roots are considered, the cubic factor in (24) can be written as $(v-$ $\left.v_{1}\right)^{2}\left(v-v_{2}\right)$, where $v_{1}$ and $v_{2}$ (both different from -1 ) can be found explicitly. Then, by Theorem $2(i i)$ (Primrose [16], pp. 7-8), we find that the curve (21) (in the limit $\alpha \rightarrow 0$ ) possesses only two asymptotes: $q=-p$ and $q=p / v_{2}$. This implies instability at large shear. Hence, long waves with small $J$ are stable if and only if the roots of (24) are all real and distinct, or, equivalently:

$$
-4 R^{3} \rho^{2}+\left(R^{4}+18 R^{2}-27\right) \rho-4 R^{3}>0 .
$$

The inequality (25) becomes a necessary condition for stability of low Richardson number flows in Holmboe's configuration (see figure 9). Based on the monotonic behaviour of the outer stability boundaries as functions of $\alpha$ in figure 8, the strict inequality in (25) seems to be a sufficient condition for stability, but its proof is nontrivial so that no conclusion on the sufficiency is drawn here.

\section{Concluding remarks}

We have proved that the basic flows proposed by Taylor [5], Goldstein [4], and Holmboe [6] can all be stable at low Richardson number, provided that certain criteria are met. These findings are relevant for two reasons: first, this observation has not been fully explored yet; second, the recourse to the theory of plane algebraic curves allows us to derive simple explicit criteria for this feature to hold.

Our theoretical results may seem at first not consistent with the literature, view that these physical configurations were originally proposed as prototypes to a better understanding of unstable processes in stratified shear flows (see the recent review by Carpenter et al. [24]). However, in the literature the stability analysis often discards the confinement of the flow by two rigid walls and relies almost exclusively on the Boussinesq approximation. These two missing ingredients may have important consequences on the stability characteristics of the flow, especially when dealing with regimes characterized by a large shear across the layers, as shown in this paper. A simplified analysis under the typical assumptions should thus be taken with caution. Further supporting evidences of this claim can be found in Barros \& Choi [13], where the wavelengths and phase speeds of Holmboe waves have been examined extensively with and without the Boussinesq approximation. 
In the limit of zero Richardson number with zero density jumps, the stability results of homogeneous shear flows are always recovered from our analysis. On the other hand, when there is stratification, we should remark that the zero-Richardson number limit due to large shear is different from the homogeneous case, as shown e.g., in figure 2(c). Nonetheless, we stress that the Boussinesq approximation always yields the homogeneous results in the the zero-Richardson number limit, but may fail to describe the stability characteristics of the flow at this regime.

We also point out that the stability feature found in this work is not aligned with what was presented by Hazel [9], when studying the effect of boundaries on "tanh" profiles for both the density and the mean horizontal velocity. In Hazel's case, as the boundaries are brought closer and closer together, we should recover Howard's stability result on the homogeneous hyperbolic tangent shear layer [25], for which no signs of instability are expected to be found for $r<1.1997$ (see figure 3 in [9]). In contrast with this situation, our flow remains mainly unstable, and stability can only be found at low Richardson number. This discrepancy seems to arise since the vertical length scale for density variation was fixed in Hazel's case as the the thickness of the fluid domain was reduced. If the thickness of the density transition layer was reduced as well in Hazel's study, our result should have been recovered although more numerical studies for continuously stratified shear flows should be performed to confirm this argument.

Finally, since there are evidences of linearly stable gravity waves in new regimes, a new long internal wave phenomenon in a stratified flow with a large shear might as well be possible, which would be interesting to explore.

\section{Acknowledgement}

This work was partially done when one of the authors (R.B.) was visiting the Instituto Nacional de Matemática Pura e Aplicada (Brazil) as a Research Fellow, and he gratefully acknowledges support for his stay. R.B. acknowledges the support of MACSI, the Mathematics Applications Consortium for Science and Industry (www.macsi.ul.ie) funded by the Science Foundation Ireland Investigator Award 12/IA/1683. Finally, R.B. would also like to thank J. F. Voloch and I. Vainsencher for helpful discussions, and for bringing plane algebraic curves to his attention. W.C. gratefully acknowledges support from the National Research Foundation of Korea funded by the Ministry of Education, Science and Technology through the World Class University (WCU) program with grant no. R31-2008-00010045-0.

\section{Appendix A. Degenerate cases for the Taylor-Goldstein configuration}

The form in which we have presented the curve equation (7) suggests some limit cases to be considered: $\gamma_{2} \rightarrow 1, \gamma_{3} \rightarrow 1, \gamma_{3} \rightarrow 0$, and $\beta_{3} \rightarrow 0$. An algebraic curve is said to be degenerate if it can split up into two or more curves. We will see below that this is precisely what happens in each one of the cases just mentioned.

Case $\gamma_{\mathbf{3}} \rightarrow \mathbf{0}$. The curve (7) reduces in this limit to

$$
\left(\alpha \operatorname{coth}(\alpha(r-1) / 2) p^{2}-1\right)\left(\beta_{2} q^{2}+p q+\gamma_{2}-1\right)=0 .
$$

The geometrical locus of the equation is the union of two vertical lines and an hyperbola with asymptotes $q=0$ and $\beta_{2} q+p=0$. When intersected by any line with slope 1 four points are obtained, thus the flow is always stable. This is not surprising since the limit corresponds precisely to the case when $\rho_{3}=\rho_{2}=0$, i.e., to the regime of surface gravity waves with constant current.

Case $\gamma_{\mathbf{3}} \rightarrow \mathbf{1}$. In this case, the curve (7) can be factorized as

$$
p\left(\beta_{1} p^{2} q+\left(\beta_{1} \beta_{2}+1-\beta_{3}\right) p q^{2}+\beta_{2} q^{3}+\left(\gamma_{2}-1\right) \beta_{1} p+\left(\gamma_{2}-1\right) q\right)=0 .
$$


We have the union between a vertical line $p=0$ and a cubic curve. The cubic has the asymptote $q=0$ and, depending on the parameters, two additional asymptotes could exist. For this to happen, the condition

$$
\left(\beta_{1} \beta_{2}+1-\beta_{3}\right)^{2}-4 \beta_{1} \beta_{2}>0,
$$

must be met. Notice that the cubic curve equation is homogeneous, and so the curve crosses the origin regardless of the physical parameters considered. Moreover, stability at low Richardson number holds if $\gamma_{2}>(r-1)^{2}$, which on the other hand implies that such feature can only occur when $r<2$.

Case $\gamma_{\mathbf{2}} \rightarrow \mathbf{1}$. This case is very similar to the one that we have just described $\left(\gamma_{3} \rightarrow 1\right)$. The curve is given by

$$
q\left(\beta_{1} p^{3}+\left(\beta_{1} \beta_{2}+\gamma_{3}\left(1-\beta_{3}\right) p^{2} q+\gamma_{3} \beta_{2} p q^{2}+\left(\gamma_{3}-1\right) p+\left(\gamma_{3}-1\right) \beta_{2} q\right)=0 .\right.
$$

and splits up into an horizontal line $q=0$ and a cubic curve. The cubic has the asymptote $p=0$ and, depending on the parameters, two additional asymptotes can exist. Stability at low Richardson number holds if $\gamma_{3}<1 /(r-1)^{2}$, and hence it can be observed for any finite value of $r$.

Case $\boldsymbol{\beta}_{\mathbf{3}} \rightarrow \mathbf{0}$. This limit corresponds to letting $\alpha$ go to infinity. For this reason, it is convenient first to rewrite $(7)$ as

$$
\frac{1}{\alpha}\left(\beta_{1} p^{2}+\gamma_{3} p q+\gamma_{3}-1\right) \frac{1}{\alpha}\left(\beta_{2} q^{2}+p q+\gamma_{2}-1\right)=\gamma_{3} \operatorname{csch}^{2} \alpha p^{2} q^{2} .
$$

Then, in the limit when $\alpha \rightarrow \infty$, we get

$$
p^{2} q^{2}=0,
$$

whose intersection with any line with slope 1 is composed by four points corresponding to two distinct real roots, each with multiplicity two. This confirms that the instability band in the diagrams of Fig. 2 is further reduced as both $J$ and $\alpha$ increase.

There is a less obvious limit that consists on letting $\beta_{1}$, or $\beta_{2}$, go to zero. This can only happen if $r=\infty$ and $\alpha=0$, in which case the curve degenerates into an algebraic curve of degree 2. More precisely, the highest degree polynomial $P_{4}(p, q)$ vanishes and the curve reduces to the hyperbola

$$
\left(\gamma_{2} \gamma_{3}-1\right) p q+\left(\gamma_{2}-1\right)\left(\gamma_{3}-1\right)=0
$$

When intersected by any line with slope 1 two points are obtained, thus the flow is always stable, regardless the shear across the layers $(c f$. figure $2(a))$.

\section{Appendix B. Remark on the Taylor configuration}

We give here attention to the case when one of the asymptotes to the curve (18) has slope 1, and thus only three intersection points between the curve and the line $q=p+\mathcal{F}$ can be found. We will see that this can only happen if $r=\infty$ and $\alpha \rightarrow 0$. Indeed, if we replace $v=1$ in (19), one obtains (after some simplifications):

$$
\left(1+\beta_{2}\right)\left(\gamma_{3}+\beta_{1}\right)-\gamma_{3} \beta_{3}=0
$$

Notice, however, that the left-hand side of the equation is greater or equal to $\left(1+\beta_{2}\right)\left(\gamma_{3}+\beta_{1}\right)-\gamma_{3}$, which in turn is equal to $\beta_{1}\left(1+\beta_{2}\right)+\gamma_{3} \beta_{2}$. Then, unless $\beta_{1}=\beta_{2}=0$, the term is strictly positive. This shows that an asymptote with slope 1 can only exist if $r=\infty$ and $\alpha \rightarrow 0$, which is precisely the scenario described in figure $5(c)$.

The curve (18) is defined in this case by

$$
\left[p^{2}+\left(\gamma_{3}-1\right) p q+\gamma_{3}-1\right]\left[\gamma_{2} q^{2}-\left(\gamma_{2}-1\right) p q+\gamma_{2}-1\right]=\gamma_{3} p^{2} q^{2},
$$


and the highest degree polynomial $P_{4}(p, q)$ simplifies to

$$
P_{4}(p, q)=p q\left[\left(1-\gamma_{2}\right) p^{2}+\left\{\gamma_{2}-\left(\gamma_{2}-1\right)\left(\gamma_{3}-1\right)-\gamma_{3}\right\} p q+\gamma_{2}\left(\gamma_{3}-1\right) q^{2}\right] .
$$

If we consider the term in the brackets in (B.1) and plug in the linear relationship (10) between $p$ and $q$, we see that the coefficient of $p^{2}$ simply vanishes. As a consequence of this, the corresponding eigenvalue equation, when written as a polynomial equation for the wave speed $c$, degenerates into a cubic equation, which explains why stability holds.

While we can call this a degenerate case, it is important to emphasize that the curve itself is not degenerate.

\section{Appendix C. Singular points}

In finding double points (also known as singular points), it is helpful to bear in mind the result that a non-degenerate curve of degree $n$ cannot have more than $(n-1)(n-2) / 2$ double points $[16,26]$. We will focus on the case when $P(p, q)=0$ is an algebraic curve of degree 4 and symmetric about the origin, as in all the three cases examined in this paper. Then, $P(p, q)$ can be expressed as $\sum_{k=0}^{4} P_{k}(p, q)$, where $P_{k}(p, q)$ is a homogeneous polynomial in $p$ and $q$ of degree $k$, with $P_{1}$ and $P_{3}$ identically zero. Thus, we may write in homogeneous coordinates

$$
P_{4}(p, q)+z^{2} P_{2}(p, q)+z^{4} P_{0}=0,
$$

whose double points (finite, or at infinity) are the solutions of $P_{p}=0, P_{q}=0, P_{z}=0$, i.e., they satisfy

$$
\begin{aligned}
P_{4, p}+z^{2} P_{2, p} & =0, \\
P_{4, q}+z^{2} P_{2, q} & =0, \\
z\left(P_{2}+2 z^{2} P_{0}\right) & =0 .
\end{aligned}
$$

Double points at infinity satisfy $z=0, P_{p}=0, P_{q}=0$, which implies that $P_{4}$ has a repeated factor. In particular, we conclude that such curves do not possess nonlinear branches at infinity, since any time a line at infinity meets the curve in two coincident points, it does so at a double point. The behavior of these curves at infinity are then described simply by the asymptotes at infinity.

Finite double points (with $z \neq 0$ ), on the other hand, are governed by the system of equations

$$
\begin{aligned}
P_{4, p}+z^{2} P_{2, p} & =0, \\
P_{4, q}+z^{2} P_{2, q} & =0, \\
P_{2}+2 z^{2} P_{0} & =0 .
\end{aligned}
$$

We will treat separately two different cases:

Case when $\boldsymbol{P}_{\mathbf{0}} \neq \mathbf{0}$. We can rewrite the system (C.1)-(C.3) by multiplying the first two equations by $2 P_{0}$. Then, by using (C.3), it follows that $(p, q)$ is a solution of

$$
\begin{aligned}
& 2 P_{0} P_{4, p}-P_{2} P_{2, p}=0, \\
& 2 P_{0} P_{4, q}-P_{2} P_{2, q}=0,
\end{aligned}
$$

which is a system of two homogeneous polynomials of degree 3 in the variables $p$ and $q$. We can now define $v \equiv p / q$ to write a system of two cubic polynomials in $v$. The cubics have a common root if the resultant vanishes. The result can then be used to find the candidate double points. 


\section{(i) Taylor-Goldstein's configuration}

We consider the curve on the $(p, q)$-plane defined by Eq. (7). One can compute the resultant of the cubic polynomials obtained from (C.4)-(C.5) and find that the candidate finite double points of the curve are solutions of:

$$
64 \beta_{1}^{2} \beta_{2}^{2} \beta_{3}^{2}\left(\gamma_{2}-1\right)^{4}\left(\gamma_{3}-1\right)^{4}{\gamma_{3}}^{2} F\left(\alpha, r, \gamma_{2}, \gamma_{3}\right)=0,
$$

where $F$ is defined by

$$
F=8 \beta_{2} \beta_{2}\left(\gamma_{2}-1\right)\left(\gamma_{3}-1\right) A_{1}\left(\beta_{1}, \beta_{2}, \beta_{3}, \gamma_{2}, \gamma_{3}\right)+B_{1}^{2}\left(\beta_{3}, \gamma_{2}, \gamma_{3}\right),
$$

with

$$
\begin{gathered}
A_{1}=1+2 \beta_{1} \beta_{2}\left(\gamma_{2}-1\right)\left(\gamma_{3}-1\right)+\gamma_{3}\left[\left(\gamma_{2}-2\right)\left(2+\left(\gamma_{2}-2\right) \gamma_{3}\right)-4 \beta_{3}\left(\gamma_{2}-1\right)\left(\gamma_{3}-1\right)\right], \\
B_{1}=1+\left[2\left(\gamma_{2}-2\right)+4 \beta_{3}\left(1-\gamma_{2}\right)\right] \gamma_{3}+\left[\left(\gamma_{2}-2\right)^{2}+4 \beta_{3}\left(\gamma_{2}-1\right)\right] \gamma_{3}{ }^{2} .
\end{gathered}
$$

We rely on extensive numerical tests to claim that $F$ is strictly positive. Hence, unless the curve is degenerate (see Appendix A), double points cannot exist. This corroborates that, in general, the curve with Eq. 7 has only two possible configurations ( $c f$. left and right panels of Fig. 1) regardless of the physical parameters. More importantly, the transition between the two scenarios is made through the existence of a double point at infinity, as the value of $\alpha$ is increased.

\section{(ii) Taylor's configuration}

The candidate finite double points of the curve on the $(p, q)$-plane defined by Eq. (18) are solutions of:

$$
16\left(\beta_{1}+1\right)^{2}\left(\beta_{2}+\gamma_{2}\right)^{2}{\beta_{3}}^{2}\left(\gamma_{2}-1\right)^{6}\left(\gamma_{3}-1\right)^{6}{\gamma_{3}}^{2} G\left(\alpha, r, \gamma_{2}, \gamma_{3}\right)=0,
$$

where $G$ is defined by

$$
G=\left(1+\beta_{2}+\beta_{1} \beta_{2}+\gamma_{2} \beta_{1}\right)^{2}-2 \gamma_{3} A_{2}\left(\beta_{1}, \beta_{2}, \beta_{3}, \gamma_{2}, \gamma_{3}\right)+B_{2}^{2}\left(\beta_{3}, \gamma_{2}, \gamma_{3}\right),
$$

with

$$
A_{2}=\left(\beta_{1}+1\right) \beta_{2}\left(1-\gamma_{2}+\beta_{3}\right)+\left(1-\gamma_{2}\right)\left(1+\gamma_{2} \beta_{1}\right)+\beta_{3}\left[\left(\beta_{1}+2\right) \gamma_{2}-1\right], \quad B_{2}=\left(\gamma_{2}-1+\beta_{3}\right) \gamma_{3} .
$$

We rely on extensive numerical tests to claim that $G$ is strictly positive. Similarly to what has been done in Appendix A for the Taylor-Goldstein configuration, it can be easily recognized that the limits $\beta_{3} \rightarrow 0, \gamma_{2} \rightarrow 1, \gamma_{3} \rightarrow 1$, and $\gamma_{3} \rightarrow 0$ all correspond to degenerate cases for Taylor's configuration. Bearing in mind that no singular points at infinity exist for this configuration, since we have shown in $\S 4.1$ that $P_{4}(p, q)$ has no repeated factors, we can state that, in general, no singular points (finite, or at infinity) exist. As a conclusion, one single configuration can exist for the curve (18).

Case when $\boldsymbol{P}_{\mathbf{0}}=\mathbf{0}$. Without loss of generality, we can assume $z=1$ and solve

$$
\begin{aligned}
P_{4, p}+P_{2, p} & =0, \\
P_{4, q}+P_{2, q} & =0, \\
P_{2} & =0 .
\end{aligned}
$$

In case $P_{2}$ is given as a product of linear factors, insert these linear relationship between $p$ and $q$ into the first two equations to get polynomial equations in one single variable. Suppose $a p+b q$ (with 
$a \neq 0$ ) divides $P_{2}$. Then, inserting the relation $p=\lambda q$, with $\lambda=-b / a$ into the first two equations yields the following system of equations

$$
\begin{aligned}
& q\left(D_{1} q^{2}+E_{1}\right)=0, \\
& q\left(D_{2} q^{2}+E_{2}\right)=0,
\end{aligned}
$$

for certain coefficients $D_{1}, D_{2}, E_{1}$, and $E_{2}$. One trivial solution is obtained when $q=0$ and $p=0$, i.e., the origin is a singular point. Other solutions may exist provided that $D_{1} E_{2}=D_{2} E_{1}$.

(iii) Holmboe's configuration

Notice that the curve equation (21) has its homogeneous polynomial $P_{2}(p, q)$ given by

$$
P_{2}(p, q)=4(\rho-1)\left(p+\lambda_{1} q\right)\left(\lambda_{1} p+q\right) .
$$

The fact that $P_{2}$ is prescribed as the product of linear factors is essential to use what was described above. Besides the origin, we can infer that the candidate finite double points of the curve are solutions of

$$
16\left(\lambda_{1}-1\right)^{2}\left(\lambda_{1}+1\right)^{2} \lambda_{3} \rho(1-\rho)=0
$$

Four limits should be examined: $\lambda_{1} \rightarrow 1, \lambda_{3} \rightarrow 0, \rho \rightarrow 0$, and $\rho \rightarrow 1$. The first is obtained only if $r=\infty$ and $\alpha \rightarrow 0$, under which (21) reduces to

$$
4 \rho(p+q)^{2}\left(p q+q^{2}-q+(\rho-1) / \rho\right)=0 .
$$

The curve is degenerate, splitting up into one (double) line and one hyperbola, and the flow is stable regardless the shear across the layers. It can be easily checked that the remaining cases produce degenerate curves as well. This said, in general, only the origin is a finite singular point.

We focus now on the non-degenerate cases and classify the possible configurations for the algebraic curve. Similarly to what was found for the elementary flow $(i)$ proposed by Taylor and Goldstein, any transition between different configurations will be made at the expense of a singular point at infinity. To find these, we only need to examine when the highest degree polynomial $P_{4}(p, q)$, given by

$$
\begin{aligned}
P_{4}(p, q)=(p+q)\left[\left(p+\lambda_{1} q\right)\left\{\left(\lambda_{1} p+q\right)\left(\left(\lambda_{2}-2\right) q+\left(\lambda_{2}+2\right) p\right)-\lambda_{3} p(p+q)\right\}-\right. \\
\left.-\rho\left(\lambda_{1} p+q\right)\left[2(p-q)\left(p+\lambda_{1} q\right)+\lambda_{3} q(p+q)\right]\right]
\end{aligned}
$$

has a repeated factor. To do that, it is convenient to define $v \equiv p / q$ and look for multiple roots of the polynomial equation $P_{4}(v, 1)=0$. It is clear that $v=-1$ is a solution. It would be a multiple root provided that $\lambda_{1} \rightarrow 1$ or $\rho \rightarrow 1$, which can be discarded, since both correspond to degenerate cases. As a result, $p+q=0$ is an asymptote to the curve (21), regardless of the physical parameters.

To inspect for other possible multiple roots, consider the cubic yield by the terms in the brackets in (C.6). As any odd degree polynomial equation, this cubic has at least one real root, thus allowing two or four asymptotes at infinity to the curve (21). The transition between the two scenarios will happen when the discriminant for the cubic vanishes. Figure 10 shows, for different values of $\rho$, when such transition occurs, and indicates that there are precisely two different configurations ( $c f$. figure 7 ).

\section{Appendix D. Symmetric instabilities under the Boussinesq approxi- mation}

One of the great advantages of using Boussinesq approximation is that the dispersion relations obtained are considerably simplified. In terms of the algebraic curves presented in this manuscript, 


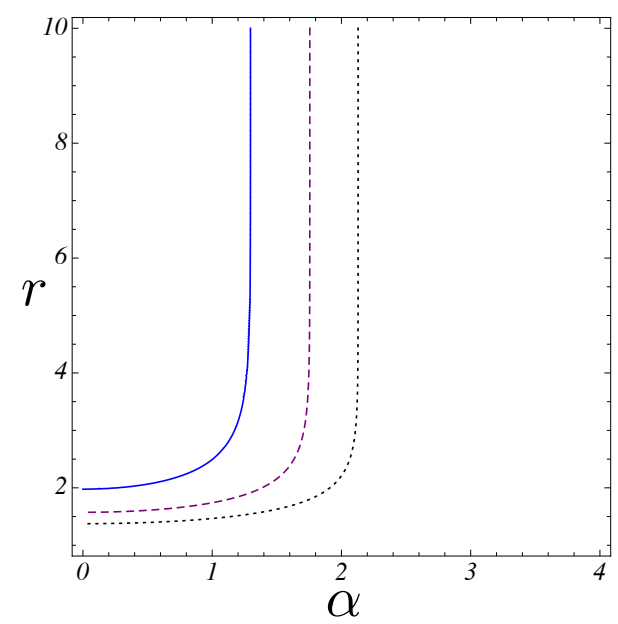

Figure 10: Transition between the two possible curve configurations for Holmboe's basic flow ( $c f$. figure 7). Different values of $\rho$ are considered: $\rho=0.998$ (full line); $\rho=0.8$ (dashed line); $\rho=0.6$ (dotted line).

the Boussinesq approximation yields extra symmetry properties. The curves preserve the symmetry about the origin, as it is the original case without approximations, and gains symmetry with respect to the line $q=p$ (and also the line $q=-p$, as a by-product). Notice that symmetry about the origin implies that the curve equation contains only even powers, or only odd powers, of $p$ and $q$ combined. If symmetry with respect to the line $q=p$ is imposed in addition, then $a_{4,0}=a_{0,4}, a_{3,1}=a_{1,3}$, and $a_{2,0}=a_{0,2}$, where $a_{i, j}$ is the coefficient of $p^{i} q^{j}$. This allows us to conclude that such curves have the general form

$$
P(p, q)=a p^{4}+b p^{3} q+c p^{2} q^{2}+b p q^{3}+a q^{4}+d p^{2}+e p q+d q^{2}+f=0 .
$$

If we rewrite the equation as

$$
P(p, q)=a\left[\left(p^{2}+q^{2}\right)^{2}-2(p q)^{2}\right]+b p q\left(p^{2}+q^{2}\right)+c(p q)^{2}+d\left(p^{2}+q^{2}\right)+e p q+f=0,
$$

we see that the curve can be expressed strictly in terms of $p^{2}+q^{2}$ and $p q$. Let $\tilde{u}=\left(u_{1}+u_{2}\right) / 2$ be the average velocity. Then, by definition (6) of $p$ and $q$, we have:

$$
p=(\tilde{u}-c-\Delta) / \sqrt{2 g h}, \quad q=(\tilde{u}-c+\Delta) / \sqrt{2 g h},
$$

with $\Delta=\left(u_{2}-u_{1}\right) / 2$. If we define $\tilde{c}$ as the wave speed relative to the mean flow, i.e., $\tilde{c}=c-\tilde{u}$, then $p^{2}+q^{2}=\left(\tilde{c}^{2}+\Delta^{2}\right) / g h$ and $p q=\left(\tilde{c}^{2}-\Delta^{2}\right) / 2 g h$. This shows that the quartic for $\tilde{c}$ obtained from (D.1) is indeed a biquadratic form. As a result, if $\tilde{c}$ is the complex wave speed of an unstable mode, then there exists an unstable model with wave speed $-\tilde{c}^{*}$, also referred to as symmetric instabilities.

\section{References}

[1] J. W. Miles, "On the stability of heterogeneous shear flows," J. Fluid Mech. 10, 496 (1961).

[2] L. N. Howard, "Note on a paper of John W. Miles," J. Fluid Mech. 10, 509 (1961).

[3] J. L. Synge, "The stability of heterogeneous liquid," Trans. Roy. Soc. Can. 27, 1 (1933).

[4] S. Goldstein, "On the stability of superposed streams of fluids of different densities," Proc. Roy. Soc. A. 132, $524(1931)$. 
[5] G. I. Taylor, "Effect of variation in density on the stability of superposed streams of fluid," Proc. Roy. Soc. A 132, 499 (1931).

[6] J. Holmboe, "On the behaviour of symmetric waves in stratified shear layers," Geophys. Publ. 24, 67 (1962).

[7] D. Zhu and G.A. Lawrence, "Exchange flow through a channel with an underwater sill," Dyn. Atmos. Oceans 24, 153 (1996).

[8] D. Zhu and G.A. Lawrence, "Holmboe's instability in exchange flows," J. Fluid Mech. 429, 391 (2001).

[9] P. Hazel, "Numerical studies of the stability of inviscid stratified shear flows," J. Fluid Mech. 51, 39 (1972).

[10] A. Alexakis, "On Holmboe's instability for smooth shear and density profiles," Phys. Fluids 17, 084103 (2005).

[11] L. N. Howard and S. A. Maslowe, "Stability of stratified shear flows," Boundary-Layer Meteorology 4, 511 (1973).

[12] S. P. Haigh and G. A. Lawrence, "Symmetric and nonsymmetric Holmboe instabilities in an inviscid flow," Phys. Fluids A 11(6), 1459 (1999).

[13] R. Barros and W. Choi, "Holmboe instability in non-Boussinesq fluids," Phys. Fluids 23, 124103 (2011).

[14] P. G. Drazin and W. H. Reid, Hydrodynamical Stability, 2nd ed. (Cambridge University Press, Cambridge, 2004).

[15] L. V. Ovsyannikov, "Two-layer "shallow water" model," J. Appl. Mech. Tech. Phys. 20, 127 (1979).

[16] E. J. Primrose, Plane Algebraic Curves (Macmillan, London, UK, 1955).

[17] A. T. Fuller, "Root location criteria for quartic equations," IEEE Trans. Automat. Contr. 26(3), 777 (1981).

[18] E. I. Jury and M. Mansour, "Positivity and nonnegativity conditions of a quartic equation and related problems," IEEE Trans. Automat. Contr. 26(2), 444 (1981).

[19] R. Barros and W. Choi, "On the hyperbolicity of two-layer flows," in Frontiers of Applied and Computational Mathematics (World Scientific, Singapore, 2008), pp. 95-103.

[20] L. Chumakova, F. E. Menzaque, P. A. Milewski, R. R. Rosales, E. G. Tabak, and C. V. Turner, "Stability properties and nonlinear mappings of two and three-layer stratified flows," Stud. Appl. Math. 122, 123 (2009).

[21] L. Rayleigh, The theory of sound, 2nd ed. (Macmillan, 1894).

[22] G. A. Lawrence, F. K. Browand, L. G. Redekopp, "The stability of a sheared density interface," Phys. Fluids A 3, 2360 (1991).

[23] J. R. Carpenter, N. J. Balmforth, and G. A. Lawrence, "Identifying unstable modes in stratified shear layers," Phys. Fluids 22, 054104 (2010).

[24] J. R. Carpenter, E. W. Tedford, E. Heifetz, and G. A. Lawrence, "Instability in stratified shear flow: review of a physical interpretation based on interacting waves," Appl. Mech. Rev. 64, 060801 (2013).

[25] L. N. Howard, "The number of unstable modes in hydrodynamic stability problems," J. Mechanique 3, 433 (1964).

[26] J. L. Coolidge, A treatise on algebraic curves, (Dover Publications, Inc., New York, 1959). 